DOI: 10.1515/awutp -2015-0008

\title{
NOTE ON THE SCHRÖDINGER EQUATION
}

\author{
C. Bizdadea, M. M. Bârcan, I. Negru, S. O. Saliu
}

Department of Physics, University of Craiova, 13 Al. I. Cuza Street, Craiova 200585, Romania

\section{Article Info \\ Received: 28 December 2011 \\ Accepted: 19 January 2012}

Keywords: Lagrangian and Hamiltonian formalisms, Schrödinger equation.

\begin{abstract}
A second-order formalism leading to an equation describing the same dynamics as the Schrödinger one is developed under some compatible initial conditions.
\end{abstract}

It is well-known that the Euler-Lagrange [1] and Hamilton [2] equations are involved in many aspects of theoretical physics. On the one hand, the Schrödinger equation [3]-[4] can be derived from the first-order Lagrangian

$$
\Lambda_{0}=\frac{i \hbar}{2}\left(\psi^{*} \dot{\psi}-\dot{\psi}^{*} \psi\right)-\frac{\hbar^{2}}{2 m}\left(\partial_{i} \psi^{*}\right)\left(\partial_{i} \psi\right)-V \psi^{*} \psi
$$

On the other hand, the Hamiltonian formulation of the Schrödinger equation was involved in many applications of quantum mechanics [5]-[9].

In this paper we develop a second-order formalism leading to an equation that describes the same dynamics as the Schrödinger one under some compatible initial conditions. In the sequel, we restrict ourselves to the one-particle Schrödinger equation with a time independent potential $V(\mathbf{x})$.

From the canonical approach of (1), one infers the second-class constraints

$$
\chi \equiv \pi-\frac{i \hbar}{2} \psi^{*} \approx 0, \chi^{*} \equiv \pi^{*}+\frac{i \hbar}{2} \psi \approx 0,
$$

and the canonical Hamiltonian

$$
H_{0}(t)=\int d^{3} x\left(\frac{\hbar^{2}}{2 m}\left(\partial_{i} \psi^{*}\right)\left(\partial_{i} \psi\right)+V \psi^{*} \psi\right) .
$$

The notations $\pi$ and $\pi^{*}$ signify the canonical momenta conjugated with $\psi$, respectively $\psi^{*}$ 


$$
[\psi(\mathbf{x}, t), \pi(\mathbf{y}, t)]=\delta^{3}(\mathbf{x}-\mathbf{y})=\left[\psi^{*}(\mathbf{x}, t), \pi^{*}(\mathbf{y}, t)\right],
$$

where the symbol [,] denotes the Poisson bracket. Thus, the Hamiltonian equations of motion can be written as

$$
\dot{F}(\mathbf{x}, t)=\left[F(\mathbf{x}, t), H_{0}(t)\right]^{\bullet},
$$

where the Dirac bracket [10]-[12] takes the form

$$
\begin{gathered}
{\left[F_{1}(\mathbf{x}, t), F_{2}(\mathbf{y}, t)\right]^{\bullet}=\left[F_{1}(\mathbf{x}, t), F_{2}(\mathbf{y}, t)\right]-} \\
-\frac{i}{\hbar} \int d^{3} z\left[F_{1}(\mathbf{x}, t), \chi(\mathbf{z}, t)\right]\left[\chi^{*}(\mathbf{z}, t), F_{2}(\mathbf{y}, t)\right]+ \\
+\frac{i}{\hbar} \int d^{3} z\left[F_{1}(\mathbf{x}, t), \chi^{*}(\mathbf{z}, t)\right]\left[\chi(\mathbf{z}, t), F_{2}(\mathbf{y}, t)\right] .
\end{gathered}
$$

After eliminating the second-class constraints (the independent co-ordinates of the reduced phase-space are $\psi$ and $\psi^{*}$ ), with the help of (5) we find that the dynamics is governed by the equations of motion

$$
\dot{\psi}=\frac{i \hbar}{2 m} \partial_{i} \partial_{i} \psi-\frac{i}{\hbar} V \psi, \dot{\psi}^{*}=-\frac{i \hbar}{2 m} \partial_{i} \partial_{i} \psi^{*}+\frac{i}{\hbar} V \psi^{*},
$$

which are nothing but the Schrödinger equations for $\psi$ and $\psi^{*}$.

Now, we start with the Hamiltonian

$$
\bar{H}_{0}(t)=\int d^{3} x\left(\pi^{*}+\frac{i}{2 \hbar}\left(\frac{\hbar^{2}}{2 m} \partial_{i} \partial_{i} \psi-V \psi\right)\right)\left(\pi-\frac{i}{2 \hbar}\left(\frac{\hbar^{2}}{2 m} \partial_{i} \partial_{i} \psi^{*}-V \psi^{*}\right)\right)
$$

from which we derive the Hamilton equations ${ }^{1}$

$$
\begin{gathered}
\dot{\psi}=\pi^{*}+\frac{i}{2 \hbar}\left(\frac{\hbar^{2}}{2 m} \partial_{i} \partial_{i} \psi-V \psi\right), \\
\dot{\psi}^{*}=\pi-\frac{i}{2 \hbar}\left(\frac{\hbar^{2}}{2 m} \partial_{i} \partial_{i} \psi^{*}-V \psi^{*}\right), \\
\dot{\pi}=-\frac{i}{2 \hbar}\left(\frac{\hbar^{2}}{2 m} \partial_{i} \partial_{i}-V\right)\left(\pi-\frac{i}{2 \hbar}\left(\frac{\hbar^{2}}{2 m} \partial_{i} \partial_{i} \psi^{*}-V \psi^{*}\right)\right), \\
\dot{\pi}^{*}=\frac{i}{2 \hbar}\left(\frac{\hbar^{2}}{2 m} \partial_{i} \partial_{i}-V\right)\left(\pi^{*}+\frac{i}{2 \hbar}\left(\frac{\hbar^{2}}{2 m} \partial_{i} \partial_{i} \psi-V \psi\right)\right) .
\end{gathered}
$$

Regarding the equations (9-12) we choose the initial conditions ${ }^{2}$

\footnotetext{
${ }^{1}$ It is easy to see that the Hamiltonian (8) describes a non-degenerate system.

${ }^{2}$ It is obvious that the initial conditions (13-14) imply the relations $\psi^{*}\left(\mathbf{x}, t_{0}\right)=\psi_{0}^{*}(\mathbf{x})$, $\pi^{*}\left(\mathbf{x}, t_{0}\right)=-\frac{i}{2 \hbar}\left(\frac{\hbar^{2}}{2 m} \partial_{i} \partial_{i}-V\right) \psi_{0}(\mathbf{x})$.
} 


$$
\begin{gathered}
\psi\left(\mathbf{x}, t_{0}\right)=\psi_{0}(\mathbf{x}), \\
\pi\left(\mathbf{x}, t_{0}\right)=-\frac{i}{2 \hbar}\left(\frac{\hbar^{2}}{2 m} \partial_{i} \partial_{i}-V\right) \psi_{0}^{*}(\mathbf{x}) .
\end{gathered}
$$

Substituting (9) in (12) and (10) in (11) we derive the equations

$$
\begin{aligned}
& \frac{\partial}{\partial t}\left(\pi^{*}-\frac{i}{2 \hbar}\left(\frac{\hbar^{2}}{2 m} \partial_{i} \partial_{i} \psi-V \psi\right)\right)=0, \\
& \frac{\partial}{\partial t}\left(\pi+\frac{i}{2 \hbar}\left(\frac{\hbar^{2}}{2 m} \partial_{i} \partial_{i} \psi^{*}-V \psi^{*}\right)\right)=0,
\end{aligned}
$$

which lead to

$$
\begin{aligned}
& \pi^{*}(\mathbf{x}, t)-\frac{i}{2 \hbar}\left(\frac{\hbar^{2}}{2 m} \partial_{i} \partial_{i}-V\right) \psi(\mathbf{x}, t)=k(\mathbf{x}), \\
& \pi(\mathbf{x}, t)+\frac{i}{2 \hbar}\left(\frac{\hbar^{2}}{2 m} \partial_{i} \partial_{i}-V\right) \psi^{*}(\mathbf{x}, t)=k^{*}(\mathbf{x}),
\end{aligned}
$$

where $k(\mathbf{x})$ and $k^{*}(\mathbf{x})$ are some functions determined by the initial conditions. Writing down (17-18) for $t=t_{0}$ and using the initial conditions, we deduce the relations

$$
k(\mathbf{x})=0=k^{*}(\mathbf{x})
$$

such that (17-18) lead to

$$
\pi^{*}=\frac{i}{2 \hbar}\left(\frac{\hbar^{2}}{2 m} \partial_{i} \partial_{i}-V\right) \psi, \pi=-\frac{i}{2 \hbar}\left(\frac{\hbar^{2}}{2 m} \partial_{i} \partial_{i}-V\right) \psi^{*} .
$$

Inserting (20) in (9-10) we arrive at (7). In consequence, we have proved the next result: $c_{1}$ ) $\left(\psi(\mathbf{x}, t), \psi^{*}(\mathbf{x}, t), \pi(\mathbf{x}, t), \pi^{*}(\mathbf{x}, t)\right)$ are solutions of equations (9-12) subject to the initial conditions (13-14) if and only if $\left(\psi(\mathbf{x}, t), \psi^{*}(\mathbf{x}, t)\right)$ are solutions of equations (7) subject to the initial conditions (13).

It is easy to show that the Hamiltonian (8) comes from the non-degenerate second-order Lagrangian

$$
\bar{\Lambda}_{0}=\dot{\psi}^{*} \dot{\psi}-\frac{i}{2 \hbar} \dot{\psi}^{*}\left(\frac{\hbar^{2}}{2 m} \partial_{i} \partial_{i}-V\right) \psi+\frac{i}{2 \hbar} \dot{\psi}\left(\frac{\hbar^{2}}{2 m} \partial_{i} \partial_{i}-V\right) \psi^{*},
$$

which is different from that used in [13]. At the Lagrangian level the initial conditions (13-14) take the form

$$
\begin{gathered}
\psi\left(\mathbf{x}, t_{0}\right)=\psi_{0}(\mathbf{x}), \\
\dot{\psi}\left(\mathbf{x}, t_{0}\right)=\frac{i}{\hbar}\left(\frac{\hbar^{2}}{2 m} \partial_{i} \partial_{i}-V\right) \psi_{0}(\mathbf{x}) .
\end{gathered}
$$


Due to the fact that the Lagrangian (21) is non-degenerate the following standard result holds: $\left.c_{1}\right)\left(\psi(\mathbf{x}, t), \psi^{*}(\mathbf{x}, t)\right)$ are solutions to the Euler-Lagrange equations $\delta \bar{\Lambda}_{0} / \delta \psi=0$, $\delta \bar{\Lambda}_{0} / \delta \psi^{*}=0$ subject to the initial conditions (22-23) if and only if $\left(\psi(\mathbf{x}, t), \psi^{*}(\mathbf{x}, t), \pi(\mathbf{x}, t), \pi^{*}(\mathbf{x}, t)\right)$ are solutions of equations (9-12) in the presence of the initial conditions (13-14).

Thus, results $c_{1}$ ) and $c_{2}$ ) lead to th following conclusion: the solutions to the EulerLagrange equations $\delta \bar{\Lambda}_{0} / \delta \psi=0, \delta \bar{\Lambda}_{0} / \delta \psi^{*}=0$ subject to the initial conditions (22-23) coincide with the solutions to the equations (7) corresponding to the initial conditions (13).

\section{Acknowledgment}

The work of M. M. Bârcan was partially supported by the strategic grant POSDRU/88/1.5/S/49516, Project ID 49516 (2009), co-financed by the European Social Fund-Investing in People, within the Sectorial Operational Programme Human Resources Development 2007-2013.

\section{References}

[1] J.-L. Lagrange, Mécanique analitique, Courcier (1811)

[2] W. R. Hamilton, Phil. Trans. R. Soc. (part I), (1835) 95

[3] E. Schrödinger, Ann. der Physik, 81 (1926) 109

[4] E. Schrödinger, Phys. Rev. 28 (1926)1049

[5] G. Wentzel, Z. Phys. 38 (1926) 518

[6] H. A. Kramers, Z. Phys. 39 (1926) 828

[7] L. Brillouin , J. Phys. 7 (1926) 353

[8] J. Schwinger, Phys. Rev. 82 (1951) 914

[9] D. Bohm, Phys. Rev. 85 (1952) 166

[10] P. A. M. Dirac, Can. J. Math. 2 (1950) 129

[11] P. A. M. Dirac, Lectures on Quantum Mechanics, Academic Press, New York (1967)

[12] M. Henneaux, C. Teitelboim, Quantization of Gauge Systems, Princeton Univ. Press, Princeton, (1992)

[13] A. A. Deriglazov, Phys. Lett. A373 (2009) 3920 